\title{
Hypoxemia as a model for high altitude and cardiovascular risk reduction
}

\author{
Friedrich C. Luft $^{1}$
}

Published online: 26 February 2016

(C) Springer-Verlag Berlin Heidelberg 2016

Is high-altitude living healthy? Perhaps in wealthy Aspen, Colorado. Data from China suggest that the least healthy place to live there is in Tibet [1]. Of course epidemiological surveys have to be taken with (do I dare say it?) a grain of salt. High altitude could benefit cardiovascular function. Residents of the Himalayan valleys are uniquely adapted to their hypoxic environment in terms of pulmonary vasculature. Bruno et al. [2] inspected their systemic cardiovascular function. They studied 95 high-altitude dwellers and 64 controls. Bruno et al. [2] conducted cardiac ultrasound, flow-mediated dilation (FMD) of the brachial artery, carotid geometry and stiffness, and aortic pulse wave velocity (PWV) in their subjects. Eleven high-altitude dwellers that had reduced FMD were studied after $1 \mathrm{~h} 100 \% \mathrm{O}_{2}$ administration. High-altitude dwellers presented lower FMD and hyperemic velocity than controls, while systolic pulmonary artery pressure was higher, despite their adaptation. In multiple regression analysis performed in high-altitude dwellers, hyperemic velocity remained an independent predictor of FMD, after adjustment for baseline brachial artery diameter, room temperature and pulse pressure, explaining almost $10 \%$ of its variance. On the contrary, in controls, brachial artery diameter remained the only independent predictor of FMD, after adjustment for confounders. The $100 \% \mathrm{O}_{2}$ administration did not modify vascular variables.

The risk factors for ischemic heart disease in Tibetan highlanders have been investigated. Fujimoto et al. [3] found that although Tibetans had remarkably high hematocrit values and

Friedrich C. Luft

luft@charite.de

1 Experimental and Clinical Research Center, Max-Delbrück Center and Charité Medical Faculty, Berlin, Germany a decrease of eicosapentaenoic acid in both serum total lipids and serum phospholipid possibly due to their diet, they were considered to have a low incidence of ischemic heart disease. These positive risk factors are likely counteracted by other negative risk factors that were identified. Tibetan highlanders showed a decreased level of palmitic acid and an increased level of linoleic acid in serum phospholipids, which could protect against atherosclerosis. In a study of cardiovascular risk in indigenous Argentinean children living at high altitude, there was a substantially higher prevalence of high triglycerides and low high-density lipoprotein cholesterol, compared to similar children from Buenos Aires [4]. These results are not encouraging. For hypertension, the main cardiovascular risk factor, the epidemiological data at altitude also do not inspire confidence. For instance, Mingii et al. [5] studied the relationship between altitude and the prevalence of hypertension in Tibet. In their investigation, a scatter plot of altitude against overall prevalence revealed a direct correlation. Metaregression analysis revealed a $2 \%$ increase in the prevalence of hypertension with every $100 \mathrm{~m}$ increase in altitude. The locations and socioeconomic status of subjects affected the awareness and subsequent treatment and control of hypertension. Thus, an immediate argument that living at high altitude leads to cardiovascular bliss is not universally convincing.

Nonetheless, Mortimer et al. [6] reported a reduction in coronary heart disease mortality in male New Mexicans residing above $1220 \mathrm{~m}$ in order of ascending altitude, but not in women. The Swiss make the same high-altitude health claim and they chivalrously included women; however, perhaps it was the cheese [7]. Rodent studies have been equivocal. Chronic hypoxia accelerated the development of atherosclerosis in apoE gene-deleted (apoE -/-) mice, along with increased reactive $\mathrm{O}_{2}$-production and activated matrix metalloproteinase-9 (MMP-9) in the aorta according to Nakano et al. [8]. In any event, chronic hypoxia-related effects 
should involve hypoxia-inducible factor 1 -alpha (HIF-1 $\alpha$ ). HIF-1 responds to systemic oxygen levels by undergoing conformational changes and associates with hypoxia-response element (HRE) regions of promoters within hypoxia-responsive genes to induce transcription. Oxygen levels especially affect HIF- $1 \alpha$ stability, subcellular localization, and its transcriptional activity. The alpha subunit forms a heterodimer with the beta subunit. Under normoxic conditions, phosphorylated Von Hippel-Lindau (pVHL)-mediated ubiquitin protease pathway rapidly degrades HIF- $1 \alpha$; however, under hypoxia, HIF- $1 \alpha$ protein degradation is prevented and HIF- $1 \alpha$ levels accumulate to associate with HIF-1 $\beta$ to exert transcriptional roles on target genes. HIF-1 induces transcription of more than 60 genes, involved in angiogenesis, erythropoiesis, cell proliferation, iron metabolism, and inflammation. Indeed, antiinflammatory mechanisms have been described in apoE -/mice involving decreased interferon-gamma (INF- $\gamma$ ) expression $[9,10]$ and resultant increase in the anti-inflammatory cytokine, interleukin-10 (IL-10).

In this issue, Kang et al. [11] have revisited the "altitude protects from atherosclerosis" hypothesis. They exposed apoE -/- mice long-term to a fraction inspired oxygen percentage $\left(\mathrm{FiO}_{2}\right)$ of $10 \%$ (sea level is $\left.20.9 \% \mathrm{FiO}_{2}\right)$. This degree of hypoxia corresponds to an altitude of approximately $5000 \mathrm{~m}$. Five-to-six week-old female mice were studied and were kept at this $\mathrm{FiO}_{2}$ until 22-23 weeks of age (middle age). Apparently, female apoE -/- mice develop a greater plaque burden than their male counterparts [12]. The apoE -/- mice allow a good assessment of plaque in the aorta and indeed, plaque area was markedly reduced. Kang et al. [11] found a brisk upregulation of IL-10 under the hypoxemic conditions. VEGF expression also increased. Hypoxemia had no reducing effect on lipid levels, blood pressure, or other obvious atherosclerotic factors; as a matter of fact, total cholesterol and triglycerides increased with hypoxemia (similar to the Argentinian children). Reassuring is the fact that hypoxemic mice increased their hematocrit values to $60 \mathrm{vol} \%$ compared to normoxemic mice. The authors pursued their protective candidate, IL-10, in several ways. Four HRE consensus sequences were identified and chromatin immunoprecipitation was performed. But, the proof of the pudding was the inclusion of IL-10 -/- mice. These mice were bred into the apoE -/strain to produce double knockout mice. The double knockout mice were not protected by hypoxemia, in terms of aortic plaque burden. Measurements of IFN- $\gamma$ showed loss of suppression by hypoxemia in the double knockout mice. The authors present a nice story in a technically well-done study. A schematic diagram is given in Fig. 1.

Perhaps a nice control for this apoE -/- mice experiment would be to take the mice to La Rinconada, Peru that resides at $5100 \mathrm{~m}$. An alternative could be Wenquan, Tibet at $4870 \mathrm{~m}$. But, then again, the experiments of Kang et al. [11] were conducted under normobaric hypoxia (sea level atmospheric

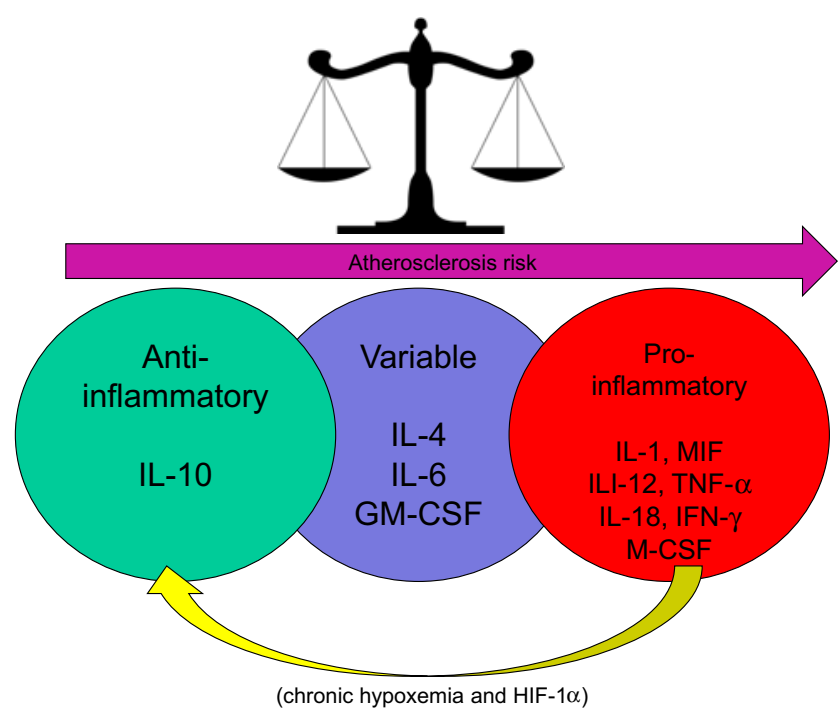

Fig. 1 Others have categorized cytokines into pro-atherogenic, antiatherogenic, and variable, that is, "not so sure" [10]. Kang et al. [11] suggest that chronic hypoxemia via INF- $\gamma$ influences the antiinflammatory IL-10 to protect against atherosclerosis in apoE -/- mice

pressure $760 \mathrm{mmHg}$ ), which is not quite the same as hypobaric hypoxia in the real world. Another aspect the authors might have considered is what the effects of hypoxemiainduced increase in alveolar ventilation could have had on their models [13]. A rough clinical estimate could be that at $\mathrm{FiO}_{2}$ of $10 \%$, the oxygen saturation would have been about $75 \%\left(\mathrm{PaO}_{2} 40 \mathrm{mmHg}\right)$, about the same as normal mixedvenous blood. The $\mathrm{PaCO}_{2}$ could have been about $25 \mathrm{mmHg}$, instead of the expected normal value of $40 \mathrm{mmHg}$, resulting in a chronic respiratory alkalosis. Reporting arterial blood-gas measurements would have been a nice touch in this study.

Finally, the authors' findings will no doubt interest the "fitness" crowd. Adaptation to living or training in hypoxic environments (altitude training) continues to gain interest from sport scientists and endurance athletes. Few persons will move to La Rinconada, Peru on the basis of the data presented by Kang et al. [11]. However, fitness lovers have alternatives including normobaric hypoxia training centers. Bonetti and Hopkins presented the first meta-analytic review of the effects on performance and related physiological measures following adaptation to six protocols of natural or artificial hypoxia: live-high train-high (LHTH), live-high train-low (LHTL), artificial LHTL with daily exposure to long $(8-18 \mathrm{~h})$ continuous, brief $(1.5-5 \mathrm{~h})$ continuous or brief $(<1.5 \mathrm{~h})$ intermittent periods of hypoxia, and artificial live-low train-high (LLTH) [14]. The analysis focused on aerobic performance so I will not dwell on it further. Nonetheless, intermittent hypobaric hypoxia (IHH) has been investigated in the venerable apoE -/- mice. Jiang et al. [15] exposed apoE -/- mice to either control conditions or IHH for 8 weeks. Mice in the IHH group were exposed to a hypobaric chamber that replicated the hypobaric hypoxia conditions found at $4000 \mathrm{~m}$ altitude for 
$8 \mathrm{~h}$ a day. IHH-exposed mice did not significantly differ from control mice in plasma lipid levels, including triglyceride, total cholesterol, low-density lipoprotein, and high-density lipoprotein. The hematoxylin and eosinstained sections of the aortic root showed similar plaque size between the groups. However, IHH-treated mice exhibited significantly decreased plaque collagen content, a feature of atherosclerotic plaque stability. Additionally, matrix metalloproteinase (MMP)-9 protein expression was significantly increased, whereas tissue inhibitor of MMP (TIMP)-2 expression was decreased. The authors suggested that IHH could promote atherosclerotic plaque instability. Kang et al. [11] could analyze the nature of their plaques in more detail. Perhaps plaque reduction was canceled out by plaque instability. Thus, the business of high-altitude and heart (cardiovascular) disease remains a bit muddled; the current results are interesting nonetheless.

Respectfully,

Friedrich C. Luft

\section{References}

1. Zhou M, Wang H, Zhu J, Chen W, Wang L, Liu S, Li Y, Wang L, Liu Y, Yin P et al (2016) Cause-specific mortality for 240 causes in china during 1990-2013: a systematic subnational analysis for the global burden of disease study 2013. Lancet 387:251-272

2. Bruno RM, Cogo A, Ghiadoni L, Duo E, Pomidori L, Sharma R, Thapa GB, Basnyat B, Bartesaghi M, Picano E et al (2014) Cardiovascular function in healthy himalayan high-altitude dwellers. Atherosclerosis 236:47-53

3. Fujimoto N, Matsubayashi K, Miyahara T, Murai A, Matsuda M, Shio H, Suzuki H, Kameyama M, Saito A, Shuping L (1989) The risk factors for ischemic heart disease in Tibetan highlanders. Jpn Heart J 30:27-34
4. Hirschler V, Maccallini G, Aranda C, Molinari C, San Antonio de los Cobres Study G (2012) Dyslipidemia without obesity in indigenous argentinean children living at high altitude. J Pediatr 161: 646-651 e641

5. Mingji C, Onakpoya IJ, Perera R, Ward AM, Heneghan CJ (2015) Relationship between altitude and the prevalence of hypertension in tibet: a systematic review. Heart 101:1054-1060

6. Mortimer EA Jr, Monson RR, MacMahon B (1977) Reduction in mortality from coronary heart disease in men residing at high altitude. NEJM 296:581-585

7. Faeh D, Gutzwiller F, Bopp M, Swiss National Cohort Study Group (2009) Lower mortality from coronary heart disease and stroke at higher altitudes in Switzerland. Circulation 120:495-501

8. Nakano D, Hayashi T, Tazawa N, Yamashita C, Inamoto S, Okuda N, Mori T, Sohmiya K, Kitaura Y, Okada Y et al (2005) Chronic hypoxia accelerates the progression of atherosclerosis in apolipoprotein e-knockout mice. Hypertension Res 28:837-845

9. Ben-Shoshan J, Afek A, Maysel-Auslender S, Barzelay A, Rubinstein A, Keren G, George J (2009) Hif-1 alpha overexpression and experimental murine atherosclerosis. Arterioscler Thromb Vasc Biol 29:665-670

10. Kleemann R, Zadelaar S, Kooistra T (2008) Cytokines and atherosclerosis: a comprehensive review of studies in mice. Cardiovasc Res 79:360-376

11. Kang JG, Sung HJ, Amar MJ, Pryor M, Remaley AT, Allen MD, Noguchi AC, Springer DA, Kwon J, Chen J et al (2016) Low ambient oxygen prevents atherosclerosis. J Mol Med. doi:10. 1007/s00109-016-1386-3

12. Caligiuri G, Nicoletti A, Zhou X, Tornberg I, Hansson GK (1999) Effects of sex and age on atherosclerosis and autoimmunity in apoedeficient mice. Atherosclerosis 145:301-308

13. West JB, American College of Physicians, American Physiological Society (2004) The physiologic basis of high-altitude diseases. Ann Intern Med 141:789-800

14. Bonetti DL, Hopkins WG (2009) Sea-level exercise performance following adaptation to hypoxia: a meta-analysis. Sports Med 39: $107-127$

15. Jiang S, Jin F, Li D, Zhang X, Yang Y, Yang D, Li K, Yang Y, Ma S (2013) Intermittent hypobaric hypoxia promotes atherosclerotic plaque instability in apoe-deficient mice. High Alt Med Biol 14: $175-180$ 